\title{
VIBRATION ISOLATION SYSTEM USING ZERO-POWER MAGNETIC SUSPENSION
}

\author{
Takeshi MIZUNO \\ Department of Mechanical Engineering, Saitama University \\ Shimo-Okubo 255, Saitama 338-8570, Japan \\ Phone: ++ 81488583455, Fax: ++81 488562577 \\ E-mail:mizar@mech.saitama-u.ac.jp
}

\begin{abstract}
A new vibration isolation system is proposed which uses zero-power magnetic suspension. Since a zero-power system behaves as if it has a negative stiffness, combining it with a normal spring can generate infinite stiffness against disturbances on the isolation table. It enables the system to have good performance both in reducing vibration transmitted from ground and in suppressing direct vibration. The basic characteristics of the proposed system are studied both analytically and experimentally. Copyright (C) 2002 IFAC
\end{abstract}

Keywords: magnetic suspension, magnetic bearings, active control, vibration damper, springs, accelerometers.

\section{INTRODUCTION}

Demands for high-performance vibration isolation systems have been increasing in various scientific and industrial fields such as high-precision measuring and semiconductor manufacturing. There are two kinds of vibration to be reduced by vibration isolation system. One is vibration transmitted from ground through suspension (spring). The other is vibration caused by disturbances acting on the isolation table (direct vibration). Lower stiffness of suspension is better for reducing the former while higher stiffness is better for suppressing the latter. A trade-off between them is inevitable in conventional passive-type vibration isolation systems so that their performances are limited.

Active-type vibration isolation systems do not have such limitations in principle (Yasuda and Ikeda, 1993). However, they need high-performance sensors such as servo-type accelerometers so that active-type systems tend to be costly, roughly speaking, nearly ten times as expensive as passive ones. It is a critical obstacle to increasing their applications.

This research will present a new approach to breaking through the trade-off. The proposed vibration isolation system uses a zero-power magnetic suspension system. Since a zero-power system behaves as if it has a negative stiffness, infinite stiffness against disturbances on the isolation table can be achieved by combining it with a normal spring. It enables the system to have good characteristics both in reducing vibration transmitted from ground and in suppressing direct vibration. It is to be noted that zero-power magnetic suspension systems usually need only relative-displacement sensors that cost far less than servo-type accelerometers.

This paper is organized as follows. First, the zeropower control in magnetic suspension systems is briefly described. Second, a basic configuration of the proposed vibration isolation system is shown. Third, the fundamental properties of the system are analytically studied. Fourth, experimental results are presented to demonstrate the effectiveness of the proposed method of generating infinite stiffness.

\section{ZERO-POWER MAGNETIC SUSPENSION}

\subsection{Basic Model}

The zero power control has been used in such magnetic suspension systems as momentum wheels for spacecraft stabilization (Sabnis et al., 1975) and 
carrier systems in clean room (Morishita et al., 1989) because of its power-saving characteristics. It uses a hybrid magnet consisting of an electromagnet and a permanent magnet. The electromagnet is controlled for the steady deviation of the coil current to converge to zero. As a result, the air gap length is maintained so that the attractive force by the permanent magnet balances the other static forces acting on the suspended object.

Figure 1 shows a single-degree-of-freedom-ofmotion model for analysis (Mizuno and Takemori, 2000). The suspended object with mass of $m$ is assumed to move only in the vertical direction translationally. The equation of motion is given by

$$
m \ddot{x}(t)=k_{s} x(t)+k_{i} i(t)+f_{d}(t),
$$

where $x$ : displacement of the suspended object, $k_{s}$, $k_{i}$ : gap-force and current-force coefficients, $i$ : control current, $f_{d}$ : disturbance acting on the suspended object.

The transfer function representation of the dynamics described by Eq. (1) becomes

$$
X(s)=\frac{1}{t_{o}(s)}\left(a_{23} I(s)+d_{0} F_{d}(s)\right),
$$

where each Laplace-transformed variable is denoted by its capital, and

$$
\begin{gathered}
t_{o}(s)=s^{2}-a_{21}, \\
a_{21}=\frac{k_{s}}{m}, \quad a_{23}=\frac{k_{i}}{m}, \quad d_{0}=\frac{1}{m} .
\end{gathered}
$$

\subsection{Zero-Power Control}

The zero-power control operates to accomplish

$$
\lim _{t \rightarrow \infty} i(t)=0 \quad \text { for stepwise disturbances. }
$$

In discussing the zero-power control, therefore, the disturbance should be considered to be stepwise.

$$
F_{d}(s)=\frac{F_{0}}{s} .
$$

When linear control law is applied, the control input is generally represented as

$$
I(s)=-\frac{h(s)}{g(s)} X(s),
$$

where $g(s)$ and $h(s)$ are coprime polynomials in $s$. It is assumed in the following that they are selected to stabilize the closed-loop system.

Substituting Eq.(6) into Eq.(2) gives

$$
X(s)=\frac{g(s)}{t(s)} d_{0} F_{d}(s),
$$

where
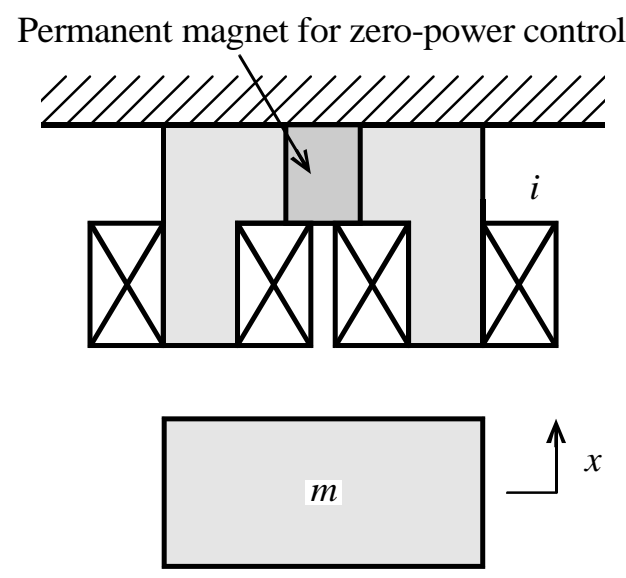

Fig.1. Basic mode of zero-power magnetic suspension

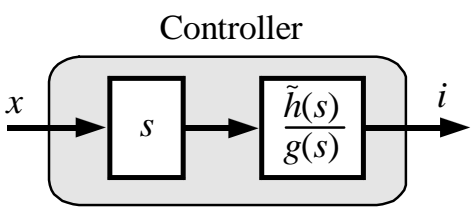

Fig.2. General form of zero-power controller

$$
t(s)=\left(s^{2}-a_{21}\right) g(s)+a_{23} h(s) .
$$

Substituting Eqs. (5) and (7) into Eq.(6) gives

$$
I(s)=-\frac{h(s)}{t(s)} d_{0} \cdot \frac{F_{0}}{s} .
$$

To achieve the control object (4), $h(s)$ must satisfy

$$
h(s)=s \tilde{h}(s),
$$

where $\tilde{h}(s)$ is an appropriate polynomial. Figure 2 shows a general form of zero-power controller (Mizuno and Takemori, 2000).

Assuming that $h(s) / g(s)$ is restricted to be proper, the minimal-order compensator achieving zeropower control and assigning the closed-loop poles arbitrarily can be represented as

$$
I(s)=-\frac{s\left(\tilde{h}_{2} s+\tilde{h}_{1}\right)}{s^{2}+g_{1} s+g_{0} s} X(s),
$$

(Mizuno and Takemori, 2000). The poles to be assigned being given, the coefficients $g_{i}{ }^{\prime} s$ and $h_{i}{ }^{\prime} s$ are determined uniquely.

\subsection{Negative Stiffness}

A unique characteristic of the zero-power control system is that it behaves as if it has a negative stiffness. When an external force is applied to the mass in common mass-spring systems, the mass moves to the direction of the applied force as shown in Fig.3a. In the zero-power controlled system, the suspended object moves to a new equilibrium position located in the direction opposite to the 
applied force as shown in Fig.3b. This characteristic is shown analytically as follows.

In the steady states, the suspended object is maintained at a position satisfying

$$
0=k_{s} x(\infty)+k_{i} i(\infty)+F_{0} .
$$

In the zero-power control system, the coil current is controlled to converge to zero, that is,

$$
i(\infty)=0 .
$$

Therefore,

$$
x(\infty)=-\frac{F_{0}}{k_{s}} .
$$

The negative sign appearing in the right-hand side indicates that the new equilibrium position is in the direction opposite to the applied force.

When a stepwise force is applied to the suspended object, it first moves in the same direction as the applied force and then to the new equilibrium position given by (14) (Mizuno and Takemori, 2000). The zero-power magnetic suspension system, therefore, has negative stiffness in a static sense.

\section{VIBRATION ISOLATION SYSTEM}

\subsection{Concept}

First it will be shown that infinite stiffness can be realized by connecting a normal spring with a spring that has negative stiffness. When two springs with spring constants of $k_{1}$ and $k_{2}$ are connected in series as shown by Fig.4, the total stiffness $k_{c}$ is given by

$$
k_{c}=\frac{k_{1} k_{2}}{k_{1}+k_{2}} .
$$

This equation shows that the total stiffness becomes lower than that of each spring when normal springs are connected. However, if one of the springs has negative stiffness that satisfies

$$
k_{1}=-k_{2}
$$

the resultant stiffness becomes infinite, that is

$$
\left|k_{c}\right|=\infty \text {. }
$$

This research applies this principle of generating high stiffness to vibration isolation systems for direct disturbance acting on the isolation table.

\subsection{System Configuration}

Figure 5 shows the configuration of one of the proposed vibration isolation systems. A middle mass $m_{1}$ is connected to the base through a spring $k_{1}$ and a damper $c_{1}$ that work as a conventional vibration isolator. An electromagnet for zero-power magnetic suspension is fixed to the middle mass. The part of an isolation table $m_{2}$ facing the electromagnet is

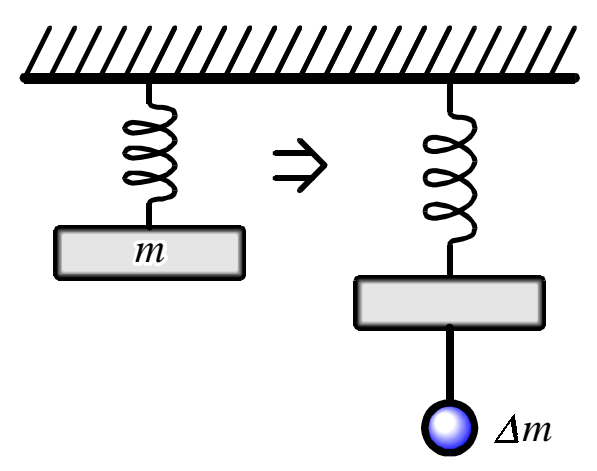

(a) Normal spring

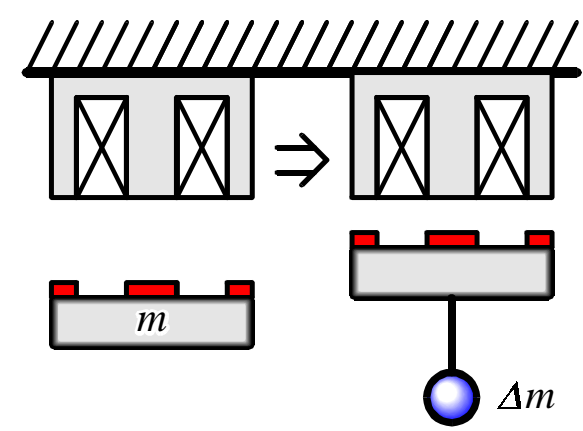

(b) Zero-power magnetic suspension system

Fig.3. Comparison of zero-power the magnetic suspension system with a normal spring.

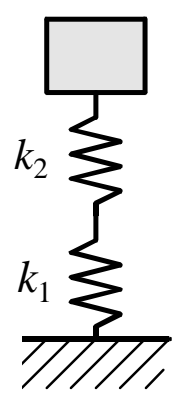

Fig.4. Series springs

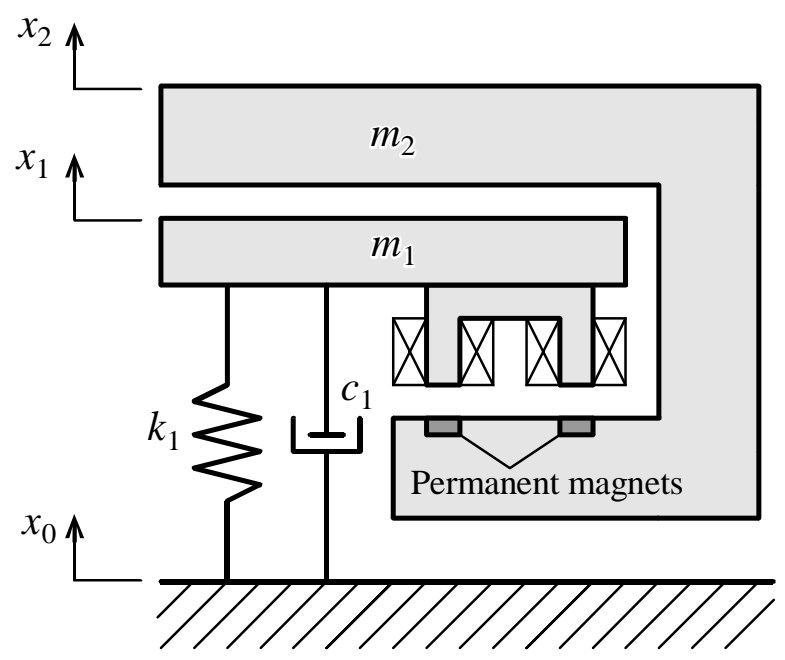

Fig.5. Vibration isolation system using zero-power magnetic suspension 
made of soft iron material confining magnetic fields produced by permanent magnets for zero-power control.

This system can reduce vibration transmitted from ground by setting $k_{1}$ small and at the same time have infinite stiffness against direct disturbance by setting the amplitude of negative stiffness equal to $k_{1}$. To put the latter more concretely, it is assumed that the table is subject to a downward force. Then the gap between the electromagnet and the table becomes smaller because of the zero-power control; in other words, the table would move upwards if the middle mass were fixed. Meanwhile, the middle mass moves downwards because of the increase of the electromagnetic force. The decrease of the gap is cancelled by the downward displacement of the middle mass because of the above-mentioned setting. Thus the isolation table is maintained at the same position as before.

In the following, it will be shown first analytically and then experimentally that the proposed system really has such property.

\section{ANALYSIS}

\subsection{Basic Equations}

The translation motions in the vertical direction will be treated here. The equations of motion are

$$
\begin{gathered}
m_{1} \ddot{x}_{1}=-k_{1}\left(x_{1}-x_{0}\right)-c_{1}\left(\dot{x}_{1}-\dot{x}_{0}\right)-f_{e}, \\
m_{2} \ddot{x}_{2}=f_{e}+f_{d},
\end{gathered}
$$

where $x_{0}, x_{1}, x_{2}$ : displacements of the floor, the middle mass and the isolation table, $f_{e}$ : control force produced by the electromagnet, $f_{d}$ : direct disturbance acting on the isolation table.

The control force is approximately represented by

$$
f_{e}=k_{s}\left(x_{2}-x_{1}\right)+k_{i} i .
$$

According to the discussion in Section 2.2, the control current achieving the zero-power control is generally represented by

$$
I(s)=-c_{2}(s) s\left(X_{2}(s)-X_{1}(s)\right),
$$

where

$$
c_{2}(s)=\frac{\tilde{h}(s)}{g(s)} .
$$

A formulation of $c_{2}(s)$ is given by eq.(11).

\subsection{Response to Direct Disturbance}

It assumed for simplicity that the initial values are zero. From (18) to (21), we get

$$
\begin{gathered}
X_{1}(s)=\frac{\left(c_{1} s+k_{1}\right) t_{2}(s)}{t_{c}(s)} X_{0}(s) \\
+\frac{k_{i} c_{2}(s) s-k_{s}}{t_{c}(s)} F_{d}(s) . \\
X_{2}(s)=\frac{\left(c_{1} s+k_{1}\right)\left(k_{i} c_{2}(s) s-k_{s}\right)}{t_{c}(s)} X_{0}(s) \\
+\frac{m_{1} s^{2}+\left(c_{1}+k_{i} c_{2}(s)\right) s+k_{1}-k_{s}}{t_{c}(s)} F_{d}(s),
\end{gathered}
$$

where

$$
\begin{gathered}
t_{1}(s)=m_{1} s^{2}+c_{1} s+k_{1}, \\
t_{2}(s)=m_{2} s^{2}+k_{i} c_{2}(s) s-k_{s}, \\
t_{c}(s)=t_{1}(s) t_{2}(s)+m_{2} s^{2}\left(k_{i} c_{2}(s) s-k_{s}\right) .
\end{gathered}
$$

To estimate the stiffness for direct disturbance, the direct disturbance $f_{d}$ is assumed to be stepwise, that is,

$$
F_{d}=\frac{F_{0}}{s} \quad\left(F_{0}: \text { const }\right) .
$$

When the vibration of the floor is neglected, the steady-state displacement of the table is obtained as

$$
\begin{gathered}
\frac{x_{2}(\infty)}{F_{0}}=\lim _{s \rightarrow 0} \frac{m_{1} s^{2}+\left(c_{1}+k_{i} c_{2}(s)\right) s+k_{1}-k_{s}}{t_{c}(s)} \\
=\frac{k_{1}-k_{s}}{k_{1}\left(-k_{s}\right)}=\frac{1}{k_{1}}-\frac{1}{k_{s}} .
\end{gathered}
$$

Therefore, when

$$
k_{1}=k_{s},
$$

we get

$$
\frac{x_{2}(\infty)}{F_{0}}=0
$$

Equation (31) shows that the suspension system between the isolation table and the floor has infinite stiffness statically because there is no steady-state deflection even in the presence of stepwise disturbances acting on the table.

The steady-state displacement of the middle mass and the variation of gap are obtained as

$$
\frac{x_{1}(\infty)}{F_{0}}=\frac{1}{k_{1}},
$$

$$
\frac{x_{1}(\infty)-x_{2}(\infty)}{F_{0}}=\frac{1}{k_{s}} \text {. }
$$

Equations (32) and (33) show that the middle mass moves downward and the gap decreases when a downward force acts on the isolation table $\left(F_{0}<0\right)$. It supports well the predictions on the behavior of the proposed vibration isolation system described in Section 3.2 


\section{EXPERIMENT}

\subsection{Experimental Setup}

Figure 6 is a schematic diagram of the developed apparatus for experimental study. The middle and isolation tables are guided to be in translation in the vertical direction by linear bearings. An electromagnet is fixed to the middle table corresponding to $m_{1}$ in Fig.5. Permanent magnets providing bias flux are made of $\mathrm{NdFeB}$ materials. They are built in the target iron of the isolation table instead of the electromagnet. Such configuration has an effect of widening the operation range because repulsive force can be generated between the isolation table and the electromagnet (Mizuno and Takemori, 2000). The nominal gap between the electromagnet and the permanent magnets is about $4 \mathrm{~mm}$.

The relative displacement of the middle table to the base and that of the isolation table to the middle table are detected by eddy-current gap sensors with a resolution of $1 \mu \mathrm{m}$. Designed control algorithms are implemented with a DSP-based digital controller. A second order compensator given by eq.(11) is used for realizing zero-power control in the following experiments. The control period is $100 \mu \mathrm{s}$.

\subsection{Experimental Results}

Two kinds of experiments are carried out. The first is for estimating the negative stiffness of the zeropower magnetic suspension and the other is for measuring the displacement of the isolation table for direct disturbance.

Figure 7 shows the results of the first experiment. The middle table is fixed during measurement. The

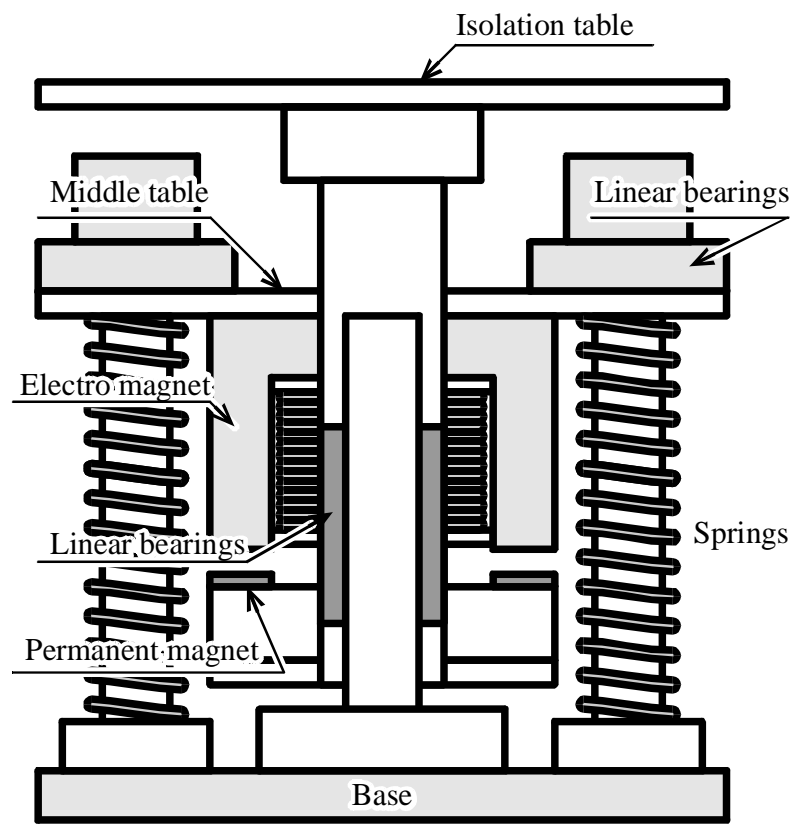

Fig.6. Experimental instrument upward displacement of the isolation table is plotted to the mass of weights added to the isolation table. The mass of the table $m$ is set to be
(a) $700[\mathrm{~g}]$,
(b) $1310[\mathrm{~g}]$,
(c) $1700[\mathrm{~g}]$.

Since the additional forces produced by weights are downward, the stiffness of the magnetic suspension is clearly negative. The relation between displacement and external force is linear when added weights are small. It is also found that the magnitude of stiffness becomes larger as the mass of the table increases.

In the second experiment, the middle table, suspended by springs, is movable and weights are added to the isolation table again. Figure 8 shows the relative displacement of the isolation table to the base. The stiffness of suspension of the middle table $k_{1}$

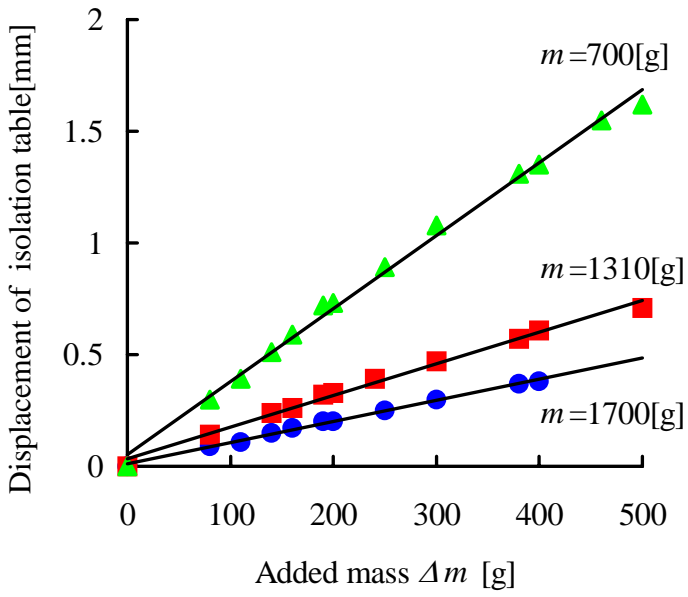

Fig.7. Load-displacement characteristics of the zeropower magnetic suspension system

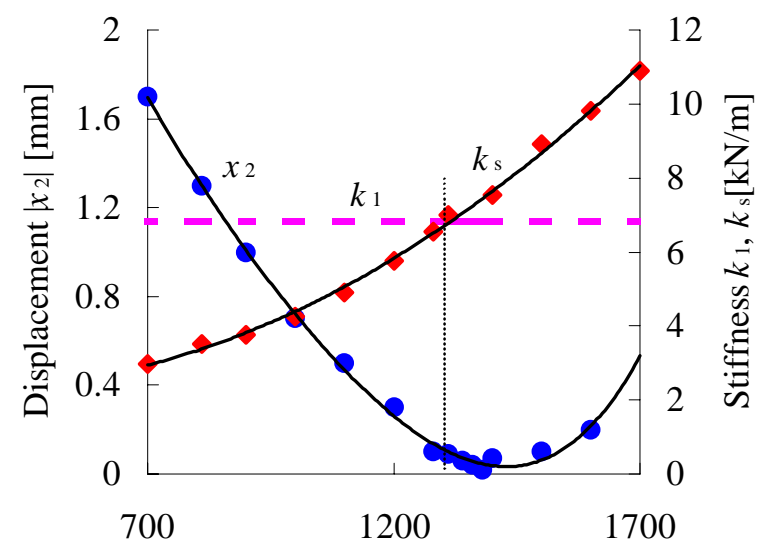

Mass of table $m[\mathrm{~g}]$

Fig.8. Displacement of the isolation table when the mass of the isolation table is varied; the stiffness of the mechanical spring and the magnetic suspension system is also shown. 
and the magnitude of the negative stiffness of magnetic suspension $k_{s}$ are also plotted in this figure. It is found from this figure that the displacement of the isolation table $\left|x_{2}\right|$ is very small when $k_{1} \cong k_{s}(m \cong 1380[g])$. The estimated stiffness between the isolation table and the base is $490[\mathrm{kN} / \mathrm{m}]$ in this region, which is about 70 times $k_{1}$ and $k_{s}(\cong 6.8[\mathrm{kN} / \mathrm{m}])$. This result demonstrates well that combining a zero-power magnetic suspension with a normal spring can generate high stiffness against static direct disturbance acting on the isolation table.

\section{CONCLUSIONS}

A new vibration isolation system using zero-power magnetic suspension was proposed. The serial connection of a normal spring and a zero-power suspension system enables the isolation system to have low stiffness for vibration from the ground and high (theoretically infinite) stiffness against direct disturbance acting on the isolation table. The principles and fundamental properties of the system were analytically studied. The effectiveness of the proposed method of realizing high stiffness against static direct disturbance was confirmed experimentally.

Mizuno (2001) has extended the method of realizing infinite stiffness to the cases where a linear actuator is used instead of a hybrid magnet in suspension.

\section{ACKNOWLEDGEMENT}

The author would like to express his thanks to Mr. R. Yoshitomi for his help with the experiments. This work is financially supported in part by a Grant-inAid for Scientific Research (B) from the Ministry of Education, Culture, Sports, Science and Technology of Japan and Electro-Mechanic Technology Advancing Foundation.

\section{REFERENCES}

Mizuno, T. and Y. Takemori (2000). A Unified Transfer Function Approach to Control Design for Virtually Zero Power Magnetic Suspension. Proc. 7th International Symposium on Magnetic Bearings, pp.45-50.

Mizuno, T. (2001). Zero-Compliance System Using Negative Stiffness. Proc. Dynamics and Design Conference 2001, 528 (in Japanese).

Morishita, M., T. Azukizawa, S. Kanda, N. Tamura, and T. Yokoyama (1989). A New Maglev System for Magnetically Levitated Carrier System. IEEE Trans. Vehicular Technology, 38, 4, pp.230-236.
Sabnis, A.V., J.B. Dendy, and F.M. Schmitt (1975). A Magnetically Suspended Large Momentum Wheel. J. Spacecraft, 12, pp.420-427.

Yasuda, M. and M. Ikeda (1993). Double-Active Control of Microvibration Isolation Systems to Improve Performances (Application of TwoDegree-of-Freedom Control). Transactions of the Japan Society of Mechanical Engineers, C, 59, 562, pp.1694-1701 (in Japanese). 